\title{
Carotid Artery and Jugular Vein Tracking and Differentiation Using Spatiotemporal Analysis
}

\author{
David Wang $^{1,2}$, Roberta Klatzky ${ }^{1}$, Nikhil Amesur ${ }^{2}$, and George Stetten ${ }^{1,2}$ \\ ${ }^{1}$ Carnegie Mellon University, Pittsburgh, PA, USA \\ ${ }^{2}$ University of Pittsburgh Medical Center, Pittsburgh, PA, USA
}

\begin{abstract}
We have derived and evaluated parameters from ultrasound images of the neck to permit a computer to automatically characterize and differentiate between the carotid artery and jugular vein at image acquisition time during vascular interventions, given manually placed seed points. Our goal is to prevent inadvertent damage to the carotid artery when targeting the jugular vein for catheterization. We used a portable $10 \mathrm{MHz}$ ultrasound system to acquire cross sectional B-mode ultrasound images of these great vessels at $10 \mathrm{fps}$. An expert user identified the vessels in the first frame by touching the vessels on the screen with his fingertip, and the computer automatically tracked the vessels and calculated a best-fit ellipse for each vessel in each subsequent frame. Vessel location and radii were further analyzed to produce parameters that proved useful for differentiating between the carotid artery and jugular vein. These parameters include relative location of the vessels, distension of the vessel walls, and consistent phase difference between the arterial and venous pulsations as determined by temporal Fourier analysis.
\end{abstract}

\section{Introduction}

The common carotid artery (CCA) and the internal jugular vein (IJV) run side-by-side in the neck, one pair on the left and one on the right. The CCA carries oxygenated blood up to the head while the IJV drains deoxygenated blood down to the heart. The right IJV is a common entry site for intravascular procedures such as central catheter line placements, hemodynamic measurements, myocardial biopsies, cardiac ablations, etc. The right IJV is chosen because its path to the right atrium is straighter than that from the left IJV. Inadvertent CCA puncture while targeting the IJV is reported to have an incidence of $2-8 \%[1,2]$ and usually results in localized hematoma. The hematoma may enlarge rapidly if the patient is coagulopathic, or if a large puncture wound is produced by the introduction of the sheath itself into the CCA. Potential negative consequences of CCA puncture include airway obstruction [3], pseudoaneurysm [4], arterio-venous fistula formation [5], retrograde aortic dissection [6], and cerebrovascular accident [7].

The introduction of B-mode ultrasound to guide IJV access has decreased the arterial puncture incidence [8]. The direction and pattern of flow from color Doppler can further help distinguish arteries from veins, but since doctors generally hold the ultrasound transducer perpendicular to the target vessels during vascular access, any slight 
angular deviation may reverse the perceived flow and make identification of the vessels ambiguous. Thus color Doppler is generally only used during pre-operative evaluation and not during the actual procedure.

We believe a semi-automated vessel identification system operating in real time during the procedure could further decrease the incidence of inadvertent CCA puncture and expedite cannulation. We expect this system to be used in conjunction with a new method of ultrasound display, the Sonic Flashlight [9], which employs a half-silvered mirror to project the image directly within the patient. By analyzing the properties of the tracked objects, it should be possible to automatically classify and graphically mark each as an artery, vein, or other tissue type. This could help prevent the operator, especially the relative novice, from accidentally puncturing the CCA.

Due in part to the presence of speckle, ultrasound is among the noisiest of all medical imaging modalities, and its anisotropy and path dependence add further difficulties for image analysis. Many researchers have developed ultrasound tracking techniques. Abolmaesumi, et al., described methods to automatically track and segment the boundary of the CCA by using the "Star" algorithm, stabilized by a temporal Kalman filter [10]. However, it requires initialization using prior knowledge about probe movement. Yeung, et al., showed that objects in sequential ultrasound images can be tracked using a deformable mesh model [11], but not in real-time. Nakayama, et al., designed an ultrasonic method of measuring arterial wall movement [12], but it requires manual outlining of the vessels and scanning in longitudinal section rather than cross section. Methods to identify anatomical targets other than vessel lumens have also been investigated. Drukker, et al., researched the use of a radial gradient index filter to automatically detect lesions in breast ultrasound [13]. Ladak, et al., developed automatic methods to segment the boundaries of the prostate [14]. Algorithms also exist for automatically locating instruments such as biopsy needles in an ultrasound image [15]. Wilson described methods using Doppler to automatically recognize the walls of a blood vessel and determine its orientation and diameter [16].

We propose to solve the problem of identifying the IJV and CCA by creating a system that will simultaneously track the vessels and provide statistical data for their identification. Our current emphasis is on identifying which of the parameters derived from our tracking algorithms best identify and distinguish between the two vessels.

We start in Section 2.1 by introducing the spokes ellipse algorithm for tracking and calculating the cross sectional elliptical radii of each vessel. In Sections 2.2 and 2.3 we explain how we calculated our parameters for differentiating the IJV from the CCA. In Section 2.4 we describe how we collected data from 16 healthy volunteers. In Section 2.5 we explain how we validated the spokes ellipse algorithm. In Section 3 we discuss the results. In Section 4 we conclude with directions for future work.

\section{Methods}

\subsection{Spokes Ellipse Algorithm}

We have developed an efficient algorithm that tracks blood vessels in real time and simultaneously calculates their elliptical radii and cross sectional area in each frame to be used in further analysis. An expert simply taps each vessel once on the screen, 
and the algorithm outlines and tracks the vessel walls in subsequent frames (Figure 1). Much like the above-mentioned "Star" algorithm, it initially draws radial lines emanating from the seed point. An intensity-based boundary detection algorithm searches for the most likely boundary along each spoke, from which an ellipse is fitted by a least squares method [17]. The cross sectional area of the vessel is approximated by the area of the ellipse. The center of the ellipse is then used as the seed point for the spokes in the next frame. By recalculating the center of the vessel and its boundaries in each frame, the vessel can be tracked in real-time, although sudden movement of the transducer may cause the tracking to be lost. This algorithm is run twice in each frame - once for the CCA and once for the IJV.

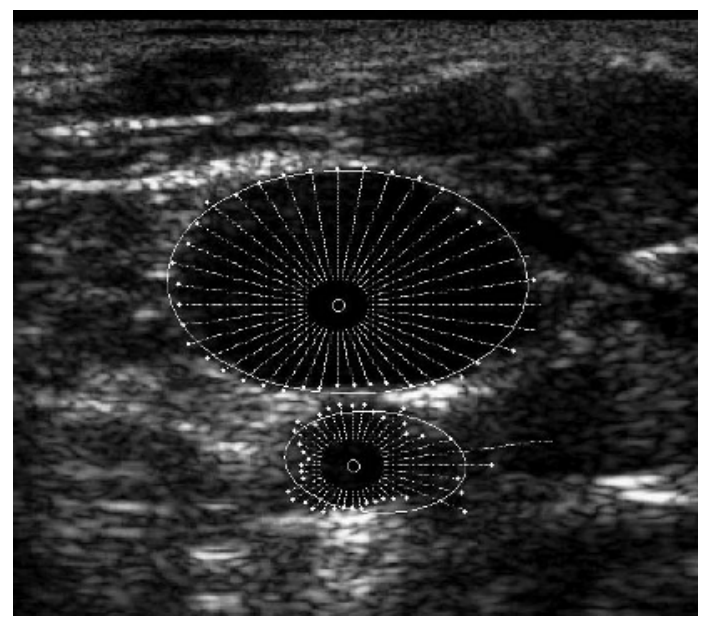

Fig. 1. Spokes ellipse algorithm applied to the IJV (top) and the CCA (bottom). Spokes grow until they reach a boundary (white dot) or a pre-set maximum length. Ellipses are fit to the dots for each vessel. Algorithm runs in real-time.

\subsection{Frequency and Phase Calculations}

By taking the Fourier transform of the series of cross sectional areas of each vessel, we can obtain the magnitude and phase at each frequency. The heart rate can be determined by identifying the frequency with the greatest magnitude between 40 and 150 cycles per minute (cpm). Similarly, the respiratory rate can be determined as the frequency with greatest magnitude in the range 10 to $30 \mathrm{cpm}$. Recall that the Fourier transform $X(\omega)$ of a signal $x(t)$ can be represented in phasor notation as

$$
X(\omega)=r(\omega) e^{j \theta(\omega)}
$$

where $r(\omega) \geq 0$ is the magnitude and $-\pi<\theta(\omega) \leq \pi$ is the phase in the complex plane. Denoting the Fourier transforms of the IJV and CCA cross-sectional areas respectively as $J(\omega)$ and $C(\omega)$, the phase difference $Q(\omega)$ between the CCA and IJV can be found from their ratio, normalized by their relative magnitudes, 


$$
Q(\omega)=\frac{r_{J}(\omega)}{r_{C}(\omega)} \frac{C(\omega)}{J(\omega)}=e^{j\left(\theta_{C}(\omega)-\theta_{J}(\omega)\right)} .
$$

This yields a unit phasor, the phase of which is the difference between the phases of the CCA and IJV at frequency $\omega$,

$$
\Delta \theta(\omega)=\theta_{C}(\omega)-\theta_{J}(\omega)
$$

Thus,

$$
\Delta \theta(\omega)=\arctan \frac{\operatorname{Im}\{Q(\omega)\}}{\operatorname{Re}\{Q(\omega)\}}
$$

Since we have a sampled Fourier transform, individual phase samples may or may not represent a consistent phase difference, so we convolve over a narrow band in the frequency domain with a normalized Gaussian smoothing filter $G(\omega, \sigma)$ as follows

$$
\Delta \tilde{\theta}(\omega)=\arctan \frac{\operatorname{Im}\{G(\omega, \sigma) * Q(\omega)\}}{\operatorname{Re}\{G(\omega, \sigma) * Q(\omega)\}}
$$

With $\sigma$ set empirically to 3 samples or $2.5 \mathrm{cpm}$ in the Fourier frequency domain. (Note that convolution can be applied to the complex number $Q(\omega)$ by applying it independently to the real and imaginary parts). A measure of phase consistency for this smoothed phase difference is

$$
\alpha(\omega)=|G(\omega, \sigma) * Q(\omega)|
$$

Random phase differences in individual samples of the Fourier transforms due to noise tend to cancel upon convolution with the Gaussian, yielding a value of $\alpha(\omega)$ near 0 , whereas a consistent phase shift over consecutive frequency samples of the Fourier transforms yields a value of $o(\omega)$ near 1 .

\subsection{Other Differentiating Factors}

Besides phase, other measures might differentiate the IJV from the CCA. In general, an artery has a thicker muscular layer than a vein. Thus the artery is stiffer while the vein is more compliant. A stiffer material emphasizes high frequencies and attenuates low frequencies. A compliant material does the opposite. We analyzed this phenomenon by calculating the slope of the linear regression that best fit the Fourier transforms from $10-250 \mathrm{cpm}$.

Since the vein is more compliant and contains blood at a lower pressure, it is less likely to assume a circular shape than an artery. We calculated the eccentricity of the two vessels as

$$
\text { eccentricity }=\sqrt{1-\frac{b^{2}}{a^{2}}}
$$

where $a$ is the semi-major axis and $b$ is the semi-minor axis. We also looked at whether the IJV is consistently closer to the skin than the CCA. 


\subsection{Experimental Protocol}

We recruited 16 healthy volunteers ages 20 to 51 . The volunteers were asked to lie supine with their heads turned to the left. The right sides of their necks were scanned with ultrasound in a standard manner. A series of digitized images was recorded from the ultrasound machine at $10 \mathrm{fps}$ for 120 seconds. Heart rates were measured by palpation before and after the scan, and the average used to estimate heart rate during the scan. Based on a preliminary data set, our exclusion criteria were IJV eccentricity > 0.8 , CCA eccentricity $>0.6$, or RMS ellipse fitting error $>0.02$ for either vessel. Excluded frames generally contained vessels that departed substantially from an elliptical form. This constituted approximately $15 \%$ of the ellipses that were fit, representing ultrasound transducer locations and orientations that would likely be deemed unsuitable for cannulation with or without our algorithm in the clinical setting. The spokes ellipse algorithm was applied to all frames collected from each subject, but only the first 500 continuous frames satisfying these inclusion criteria were used in our analysis.

\subsection{Validation of the Spokes Ellipse Algorithm}

The spokes ellipse algorithm requires two assumptions: (1) the vessel cross sections are elliptical, and (2) the algorithm-drawn ellipses are similar to manual traces of the lumen drawn by an expert. We define the similarity between two areas as

$$
\frac{\text { Area } 1 \cap \text { Area } 2}{\text { Area } 1 \cup \text { Area } 2} \times 100 \text { (the ratio expressed as a percentage) }
$$

A perfect similarity would thus have a value of $100 \%$. We randomly sampled 100 images from the 8000 frames (500 frames per subject, 16 subjects). An expert outlined the lumens of both the CCA and IJV in all 100 images. We then tested the similarity between the area of the ellipse fit to the expert tracing and (1) the expert traced area itself, and (2) the algorithm defined ellipse, to test assumptions 1 and 2, respectively.

\section{Results}

Among the 100 sampled images, there was a $91 \pm 1 \%$ similarity between the manual tracings of the lumen and the ellipses fitted to those tracings, suggesting that the cross-sectional areas of the vessels are reasonably elliptical (assumption 1). The similarity between ellipses fit to the manual tracings and the ellipses fit by the spokesellipse algorithm was $82 \pm 2 \%$ for IJV and $79 \pm 2 \%$ for CCA. Moreover, the areas of the ellipses fit to the manual tracings and algorithm-fit ellipses showed a correlation value of $r=.80$. This suggests that while the algorithm may not have fully extracted the elliptical shape defined by an expert (assumption 2), it tended to report area with a consistent bias that preserved the relative sizes of the CCA and IJV and their individual changes over time.

An expert user verified that the algorithm successfully tracked the vessels in the 500 frames that satisfied our inclusion criteria. These frames were subsequently used 
to calculate the parameters described in Sections 2.2 and 2.3 to test their reliability at differentiating between the CCA and IJV. The results are shown in Table 1.

The heart rates calculated from the CCA were not significantly different from those from the IJV. The respiratory rates also did not differ significantly. This is to be expected, since the underlying heart and respiratory rates are identical for both vessels. The fact that these heart rates are consistent with those determined by palpation during the scan (data not shown) lends further validity to our approach.

The slopes of the Fourier transform of the IJV $(-36.99 \pm 14.14)$ were significantly more negative than those of the CCA $(-16.36 \pm 2.58)$, with a p-value $<0.05$. This is consistent with the IJV being more compliant than the CCA and thus attenuating the higher frequencies.

Table 1. 95\% confidence interval for 8 parameters, derived separately (first 5 parameters) or jointly (last 3 parameters) from the CCA and IJV data. The last column shows, for the first 5 parameters, the significance level $(p)$ for a paired t-test comparing the CCA and IJV values across the sampled frames.

\begin{tabular}{|c|c|c|c|}
\hline Parameter & Vessel & 95\% Confidence Interval & $p$-val. \\
\hline \multirow{2}{*}{ Heart rate $(\mathrm{cpm})$} & CCA & $61.06 \pm 6.79$ & \multirow{2}{*}{0.40} \\
\hline & IJV & $60.91 \pm 6.88$ & \\
\hline \multirow{2}{*}{ Respiratory rate (cpm) } & $\mathrm{CCA}$ & $15.81 \pm 1.80$ & \multirow{2}{*}{0.16} \\
\hline & IJV & $17.12 \pm 2.26$ & \\
\hline \multirow{2}{*}{ Slope of Fourier transform } & $\mathrm{CCA}$ & $-16.36 \pm 2.58$ & \multirow{2}{*}{0.006} \\
\hline & IJV & $-36.99 \pm 14.14$ & \\
\hline \multirow{2}{*}{ Mean of eccentricity } & $\mathrm{CCA}$ & $0.62 \pm 0.04$ & \multirow{2}{*}{0.42} \\
\hline & IJV & $0.63 \pm 0.06$ & \\
\hline \multirow{2}{*}{ Standard deviation of eccentricity } & CCA & $0.11 \pm 0.02$ & \multirow{2}{*}{0.006} \\
\hline & IJV & $0.08 \pm 0.02$ & \\
\hline \multicolumn{2}{|c|}{ Smallest ratio of distance to skin from CCA vs. IJV } & $1.35 \pm 0.07$ & N/A \\
\hline \multicolumn{2}{|c|}{ Phase difference between CCA and IJV at heart rate } & $-1.95 \pm 0.84$ & N/A \\
\hline \multicolumn{2}{|c|}{ Phase consistency between CCA and IJV at heart rate } & $0.71 \pm 0.10$ & N/A \\
\hline
\end{tabular}

It was expected that, being more compliant, the IJV would show a greater degree of eccentricity, especially considering the pressure being applied by the ultrasound transducer. However, the respective means of eccentricity for the CCA and IJV were $0.62 \pm 0.04$ and $0.63 \pm 0.06$, a non-significant difference, indicating that the eccentricity of the vessels is not a good distinguishing feature. Eccentricity is also very dependent on the angle between image plane and direction of the vessels, so even circular vessels can appear eccentric when scanned at an angle. The slightly higher standard deviation of eccentricity for the CCA also conflicts with the expectation that the IJV would show a greater variation in eccentricity over time, and as such is suspect as a viable differentiating factor.

It was expected that the CCA would lie deeper on average than the IJV. Thus the ratio of the distance to the skin from the center of the CCA to that of the IJV should be greater than 1. Indeed, even the smallest ratios in all frames for each subject show a $95 \%$ confidence interval that excludes 1.0. However, while this observation is true when scanning at the "conventional" site, it may no longer be true when scanning at 
somewhat displaced sites on the neck. One study shows the IJV is positioned completely lateral to the CCA on the image display instead of overlapping the CCA in $8.7 \%$ of clinical scans [18]. Doctors often prefer such laterally placed IJV because CCA puncture is less likely when it is not directly under the IJV. Thus, doctors may seek scanning positions that render the vessel depth parameter invalid.

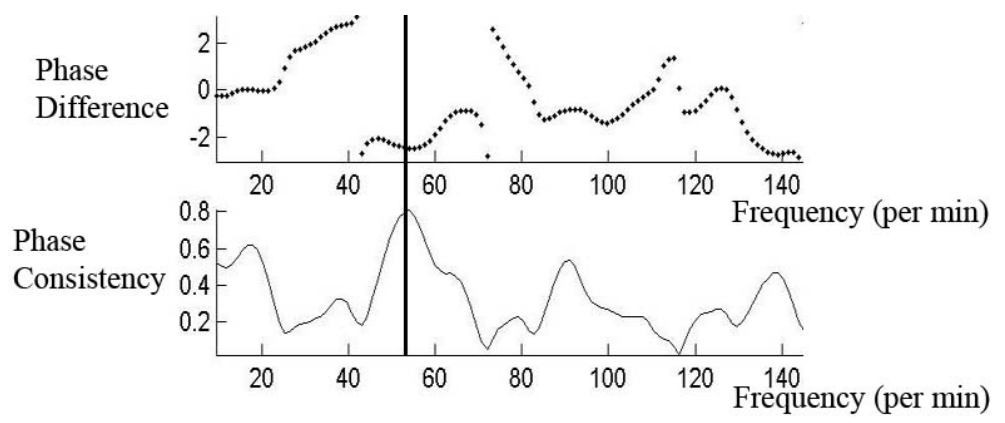

Fig. 2. (Top) Phase difference between the CCA and the IJV for subject 4 whose heart rate is around 55/min (bold vertical line). (Bottom) Phase consistency measure (see Section 2.2).

The phase difference at the heart rate has a 95\% confidence interval of $-1.95 \pm 0.84$ radians. If we had reversed the CCA and IJV in the calculation of phase difference, the $95 \%$ confidence interval would be just the negative, i.e., $1.95 \pm 0.84$ radians. Since these intervals do not include 0 nor $\pi$, it appears that phase difference can be unambiguously used to differentiate between the CCA and the IJV. In addition, phase difference shows high consistency around the frequency of the heart rate. For example, Figure 2 shows the phase (top) and consistency (bottom) for patient 4 . In the region of the heart rate $(55 \mathrm{cpm})$ the consistency approaches 1 , lending confidence to the recorded phase difference of approximately -2 radians in this region of the spectrum.

\section{Conclusions and Future Work}

We have shown in each of our 16 subjects, that the spokes ellipse algorithm could maintain tracking for at least 50 seconds (500 continuous frames) during the 120 second ultrasound scans. Although the CCA and IJV both pulsate, we have verified that the slope of the Fourier transform, the vertical locations of the vessels, and phase difference can be used individually to reliably distinguish between the vessels.

We have yet to determine what combinations of parameter weights would further optimize differentiation. Also needed is a method to reliably detect loss of tracking. While the spokes ellipse algorithm runs in real-time, the vessel classification algorithm at present incurs a lag time because the Fourier transform requires a significant number of cardiac cycles to operate. We are considering various other phase-detection methods besides Fourier that can operate within a single cardiac cycle. Future work may also incorporate color Doppler to initially identify the blood vessels, replacing manual initialization. We plan to obtain images from patients who are about to 
undergo IJV catheter insertions in the hospital to more accurately reflect the parameters in the clinical setting. Other smaller vessels in the vicinity of the CCA and IJV will also need to be accounted for. Eventually we plan to incorporate our automated vessel identification system into ongoing clinical trials of the Sonic Flashlight.

\section{References}

1. Davies MJ, Cronin KD, and Domaingue CM, "Pulmonary artery catheterization: an assessment of risks and benefits in 220 surgical patients," Anaesth Intensive Care 10:9, 1982.

2. Patel C, Laboy V, Venus B, Mathru M, Wier D, "Acute complications of pulmonary artery catheter insertion in critically ill patients," Crit Care Med, 14(3):195-7 1986 Mar

3. Kua JS, Tan IK, et al., "Airway obstruction following internal jugular vein cannulation," Anaesthesia, 52:776-80, 1997

4. Aoki H., Mizobe T., Nozuchi S., et al, "Vertebral artery pseudoaneurysm: A rare complication of internal jugular vein catheterization," Anesth Analg 75:296-298,1992

5. Gobeil F. Couture P. Girard D, Plante R, "Carotid Artery-lnternal Jugular Fistula: Another Complication following Pulmonary Artery Catheterization via the Internal Jugular Venous Route," Anesthesiology 1994, 80:23-232.

6. Applebaum RM, et al, "Transesophageal echocardiographic identification of a retrograde dissection of the ascending aorta caused by inadvertent cannulation of the common carotid artery," J Am Soc Echocardiogr 1997 Sep;10(7):749-51

7. Zaidi NA, Khan M, Naqvi HI, Kamal RS, "Cerebral infarct following central venous cannulation," Anaesthesia, 53:186-91, 1998 Feb

8. Denys BG, et al, "Ultrasound-assisted cannulation of the internal jugular vein: A prospective comparison to the external landmark-guided technique," Circulation 1993; 87:1557-62.

9. Stetten GD, Chib V, "Overlaying Ultrasound Images on Direct Vision," J Ultrasound Med 2001;20(3):235-40.

10. Abolmaesumi P, et al., "Real-Time Extraction of Carotid Artery Contours from Ultrasound Images" 13th IEEE Symposium on Computer-Based Medical Systems (CBMS'00) p. 181

11. Yeung F, et al., "Feature-Adaptive Motion Tracking of Ultrasound Image Sequences Using A Deformable Mesh" IEEE Transactions on Medical Imaging Vol. 17, No. 6, Dec 1998

12. Nakayama K, Sato S, "Ultrasonic measurement of arterial wall movement utilizing phasetracking systems," Proceedings of the 10th International Congress on Medical and Biological Engineering, Dresden, Germany, 1973:318. Abstract

13. Drukker K, et al., "Computerized lesion detection on breast ultrasound," Medical Physics, July 2002, Volume 29, Issue 7, pp. 1438-1446

14. Ladak H, et al., "Prostate boundary segmentation from 2D ultrasound images" Medical Physics, August 2000, Volume 27, Issue 8, pp. 1777-1788

15. Draper K, et al., "An algorithm for automatic needle localization in ultrasound-guided breast biopsies," Medical Physics, August 2000, Volume 27, Issue 8, pp. 1971-1979

16. Wilson LS, Dadd MJ, Gill RW, "Automatic vessel tracking and measurement for Doppler studies," Ultrasound Med Biol. 1990;16(7):645-52

17. Pilu M, Fitzgibbon A, Fisher R, "Ellipse-specific direct least-square fitting," Proceedings of the IEEE international Conference on Image Processing, IEEE Computer Society Press, Los Alamitos, CA 1996; 3:599-602.

18. Troianos CA, et al., "Internal Jugular Vein and Carotid Artery Anatomic Relation as Determined by Ultrasonography," Anesthesiology 1996; 85:43-8 